\title{
Technical Consideration for Coiling of Ruptured Proximal Posterior Inferior Cerebellar Artery Aneurysm
}

\author{
Jong Hoon Kim, M.D., Ik Chan Jeon, M.D., Chul Hoon Chang, M.D., Young Jin Jung, M.D. \\ Department of Neurosurgery, Yeungnam University Medical Center, Yeungnam University Medical School, Daegu, Korea
}

Objective : Surgical obliteration of ruptured aneurysm of the proximal posterior inferior cerebellar artery (PICA) is challenging because of limited surgical accessibility. In recent years, coil embolization is the first-choice treatment for these lesions. However, coil embolization is not always easy in ruptured PICA aneurysm owing to the variable anatomical diversity of its shapes, its relationship to the parent artery, its low incidence, and accordingly, lesser neurointerventionist experience.

Methods : The parent artery and microcatheter for easier navigation and the embolization technique for stable coiling were identified.

Results : This study aimed to identify the more appropriate approach route, microcatheter, and strategies for an easier and safer, and more durable coil embolization in the treatment of lesions in the proximal PICA.

Conclusion : Coil embolization for aneurysmal subarachnoid hemorrhage due to a ruptured proximal PICA remains a challenge, but with the appropriate coiling plan, it can be treated successfully.

Key Words : Subarachnoid hemorrhage · Proximal posterior inferior cerebellar artery · Embolization, Therapeutic.

\section{INTRODUCTION}

Subarachnoid hemorrhage (SAH) caused by aneurysmal rupture needs to be treated as soon as it is detected. When an aneurysm is not obliterated, it is likely to cause rebleeding, which may lead to clinical aggravation ${ }^{3}$. Surgical clipping and endovascular coiling are effective in preventing rebleeding and, generally, should be performed in the early phase. However, a surgical approach for acute-phase lesions in the proximal posterior inferior cerebellar artery (PICA) can be dangerous and reportedly lead to additional neurological deficits postoperatively in $\geq 50 \%$ of the cases. This is because the proximal PICA allows only for a too narrow surgical view, as it is filled with subarachnoid hematomas, is surrounded by brain stem perforation arteries, and contains major cranial nerves ${ }^{2}$. By contrast, coil embolization based on an intravascular approach can reportedly be used with relative easy to treat these lesions because it avoids the neighboring cranial nerves and subarachnoid hematomas ${ }^{1,4)}$. However, as proximal PICA may lead to an anatomical diversity of shapes when it meets its parent artery (vertebral artery [VA]), it is essential to understand the diversity in using coil embolization to treat le-

- Received : August 4, 2017 •Revised : September 21, 2017 •Accepted : October 8, 2017

- Address for reprints : Young Jin Jung, M.D.

Department of Neurosurgery, Yeungnam University Medical Center, Yeungnam University Medical School, 170 Hyunchung-ro, Nam-gu, Daegu 42415, Korea Tel : + 82-53-620-3790, Fax : + 82-53-620-3770, E-mail : sofesofe@hanmail.net

This is an Open Access article distributed under the terms of the Creative Commons Attribution Non-Commercial License (http://creativecommons.org/licenses/by-nc/4.0) which permits unrestricted non-commercial use, distribution, and reproduction in any medium, provided the original work is properly cited. 
sions in the proximal PICA and to develop diverse strategies for coil embolization. This study aimed to identify the appropriate microcatheter and strategies for coil embolization in the treatment of these lesions.

\section{MATERIALS AND METHODS}

\section{Choosing the parent artery for coil embolization}

In performing coil embolization, first consideration should be given to the positional relationship between the parent artery and the aneurysm (Fig. 1). The first step is to determine whether to move the microcatheter along the ipsilateral VA or along the opposite VA, depending on the directions of the growths of the parent artery and aneurysm. The general incidence rates of VA and PICA are shown in Fig. 1A. It is desir- able to move the microcatheter along the parent artery of the lesion when the aneurysm grows in the same direction as the VA flow (Fig. 1B and C); however, when the aneurysm grows in the opposite direction to the flow of the parent artery, the opposite VA allows the microcatheter to be placed more easily. The opposite approach could be more stable and make it easier to deliver the coil (Fig. 1D and E). As shown in Fig. 1F, a Jshaped microcatheter can be placed within the lesion through the ipsilateral VA, in which case the microcatheter is likely to come out during coil insertion because it is not strong enough to support the parent artery.

\section{Choosing the microcatheter}

Once a direction for moving the microcatheter has been decided, the next step is to choose a microcatheter (Fig. 2). The greatest consideration in deciding on the shape of a micro-
(A)

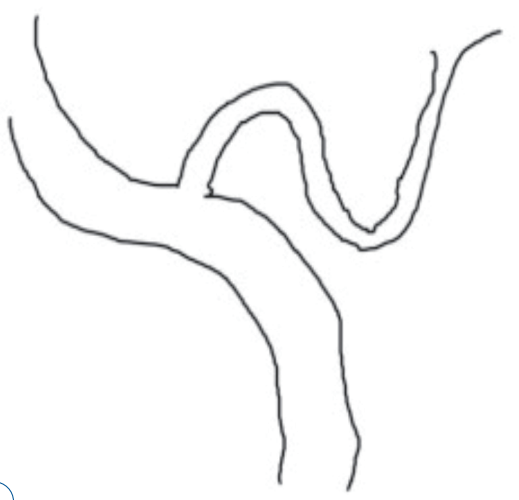

(B)

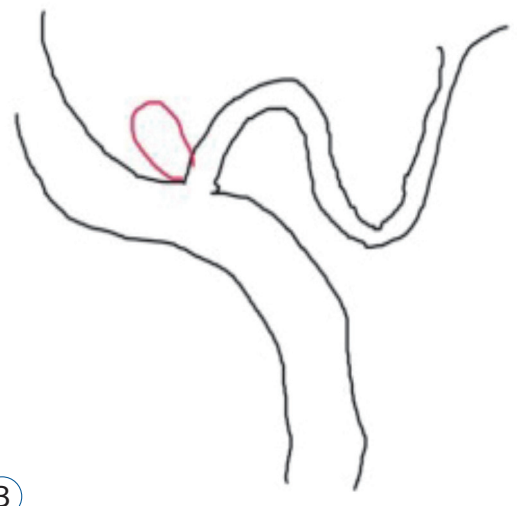

(C)
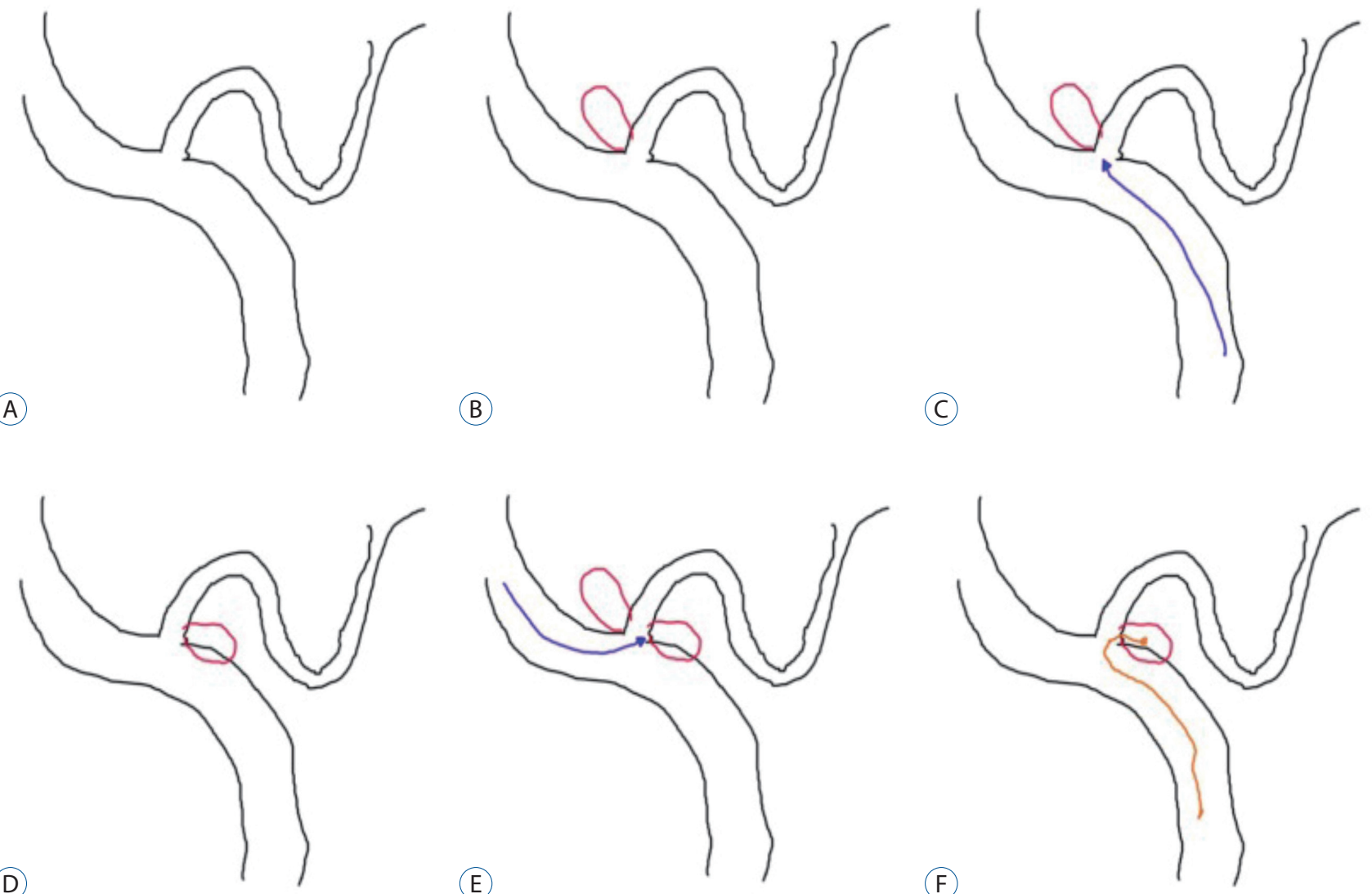

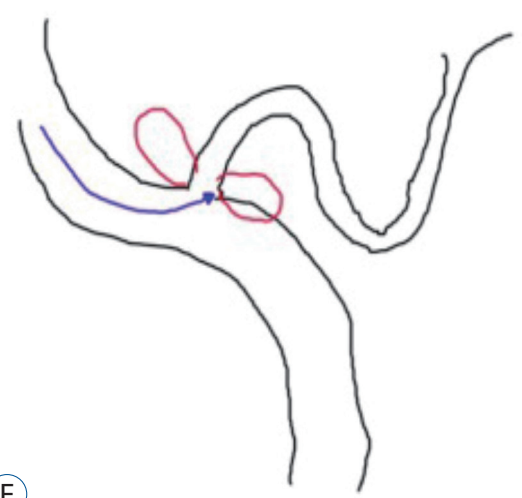

(E)

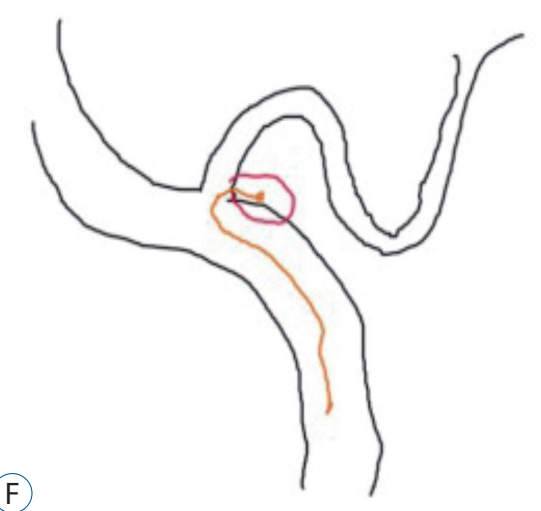

Fig. 1. General relationship between the VA and the PICA. The PICA arises from the VA and travels posterolaterally around the medulla, and makes a characteristic loop appearance (A). Usually, aneurysms grow in the direction of bloodstream flow, so superior directed aneurysms are more common among proximal PICA aneurysms (B). Thus, we can navigate the microcatheter to the aneurysm according the ipsilateral VA (C). However, sometimes, aneurysms may grow to the opposite direction (D). In these cases, the contralateral approach via the opposite VA is easier than the ipsilateral approach (E). The contralateral approach is possible with the navigation on ipsilateral side with a J-shaped microcatheter, but it is easily pushed back to the parent artery during coil delivery (F). VA : vertebral artery, PICA : posterior inferior cerebellar artery. 
catheter should be given to the agreement between the direction in which an aneurysm grows and the curvature of the parent artery. The VA generally has a curvature with an upward canvas, as shown in Fig. 2A, in most cases, and a microcatheter placed in the VA may slightly droop along its normal curvature (Fig. 2A). In such a situation, a 45 microcatheter is more likely to droop. Therefore, an S-shaped microcatheter can more favorably be placed in an aneurysm that occurs in the opposite direction to the curvature of the parent artery and help maintain a more stable form. The type of S-shaped microcatheter is slightly dependent on the angle between the parent artery and the aneurysm. As shown in Fig. 2B, it is desirable to decide on the angle of an S-shaped microcatheter depending on the angle between the parent artery and the aneurysm. Fig. 2C-E show that the form of an S-shaped microcatheter also depends on the angle between the parent artery and the aneurysm. A more acutely angled S-shaped microcatheter is necessary for larger angled aneurysm (Fig. 2F).

\section{Coil embolization technique}

Once a microcatheter is chosen, the last consideration should be given to how to obliterate the aneurysm (Fig. 3). If the neck of the aneurysm is relatively small, the aneurysm can be blocked using a single microcatheter (Fig. 3A). However, if the neck is large or if part of the aneurysm snaps at the parent artery, another microcatheter or stent can be used. When the neck is relatively small, as shown in Fig. 3A, it is easy to perform coil embolization with a single microcatheter. When the neck is large and short, as shown in Fig. 3B, using a single microcatheter to perform coil embolization can likely push out the coil into the parent artery; therefore, two microcatheters can be used to prevent the coil from coming out into the parent artery. When a large part of the parent artery is invaded by an aneurysm (Fig. 3C and D), two microcatheters can also be used to form the neck of the aneurysm in performing coil embolization. In case of fusiform dilatation (Fig. 3E-G), coil embolization can be performed by maintaining the patency of the parent artery by inserting a stent in the artery to put the coil around it.

\section{Case 1 : choosing the parent artery for the approach}

When the parent artery and aneurysm grow in the same direction, an approach through the ipsilateral VA (Fig. 4A-D) allows the microcatheter to reach the lesion with ease and leads to greater stability. When the parent artery and aneurysm grow in the opposite direction to each other (Fig. 4E-H), the microcatheter is placed in the aneurysm through the VA opposite the lesion to perform coil embolization.

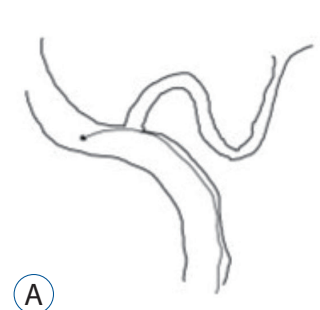

(A)

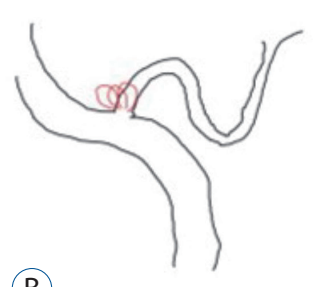

(B)

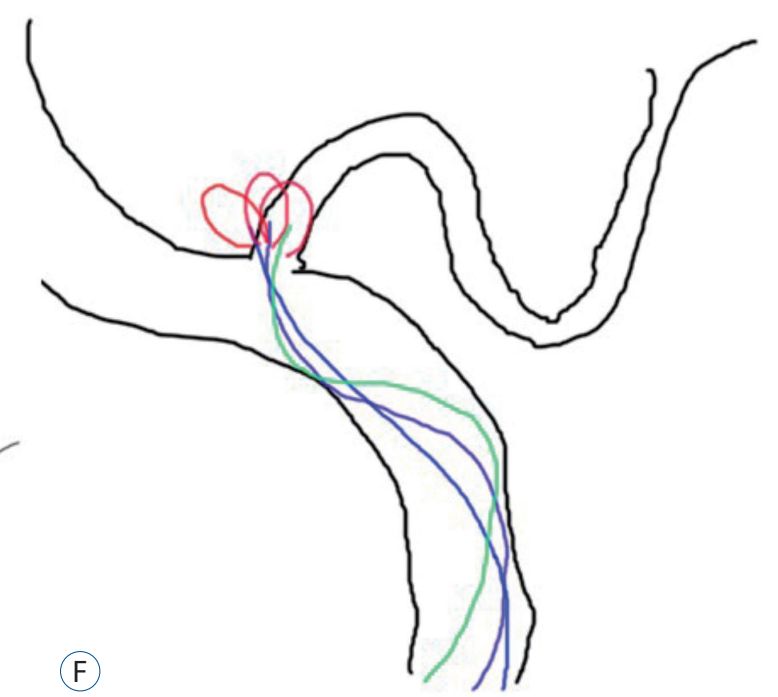

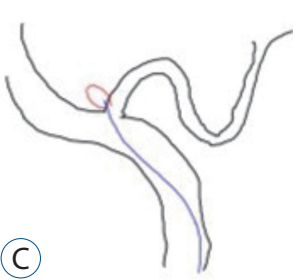

(D)
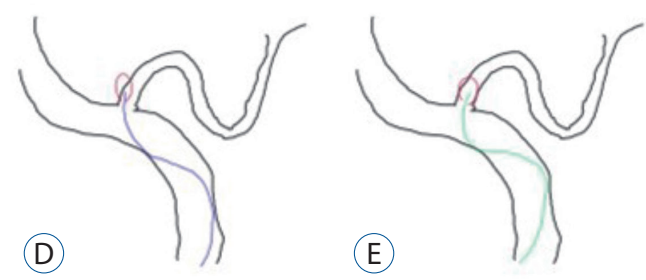

(F)

Fig. 2. Usually, the VA shows an upward curved curvature in the lateral view, and the microcatheter within the VA may slightly droop along its normal curvature (A). The aneurysm on the proximal PICA arises at a superior direction. To navigate the superior directed aneurysm, an S-shaped microcatheter could be necessary (B). The type of S-shaped microcatheter can depend slightly on the angle between the parent artery and the aneurysm. As shown in (B), it is desirable to decide on the angle of an S-shaped microcatheter depending on the angle between the parent artery and the aneurysm. C-E : Show that the form of an S-shaped microcatheter also depends on the angle between the parent artery and the aneurysm. A more acutely angled S-shaped microcatheter is necessary for larger angled aneurysms (F). VA : vertebral artery, PICA : posterior inferior cerebellar artery. 


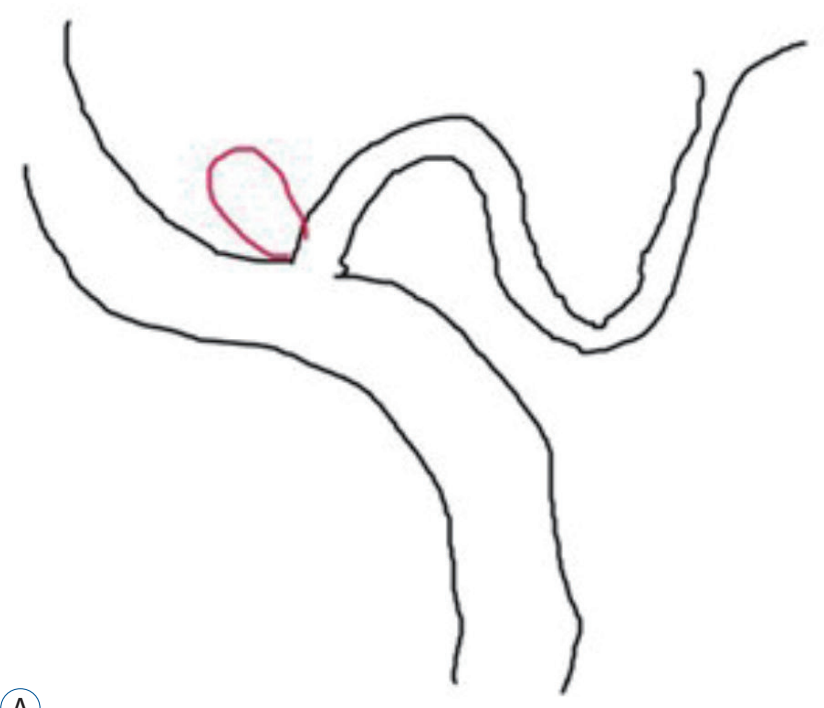

(A)

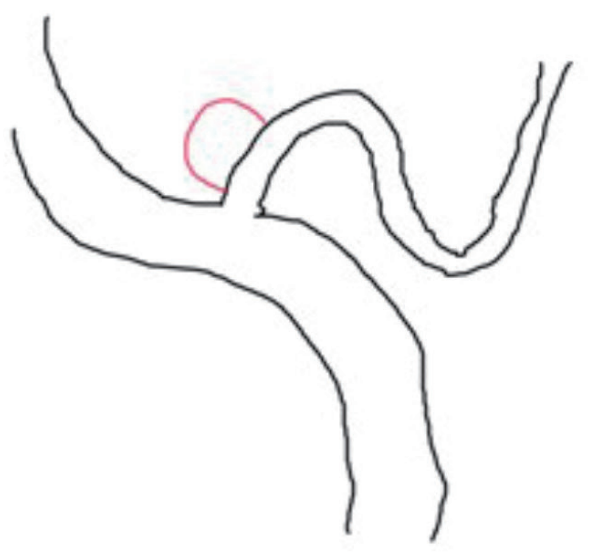

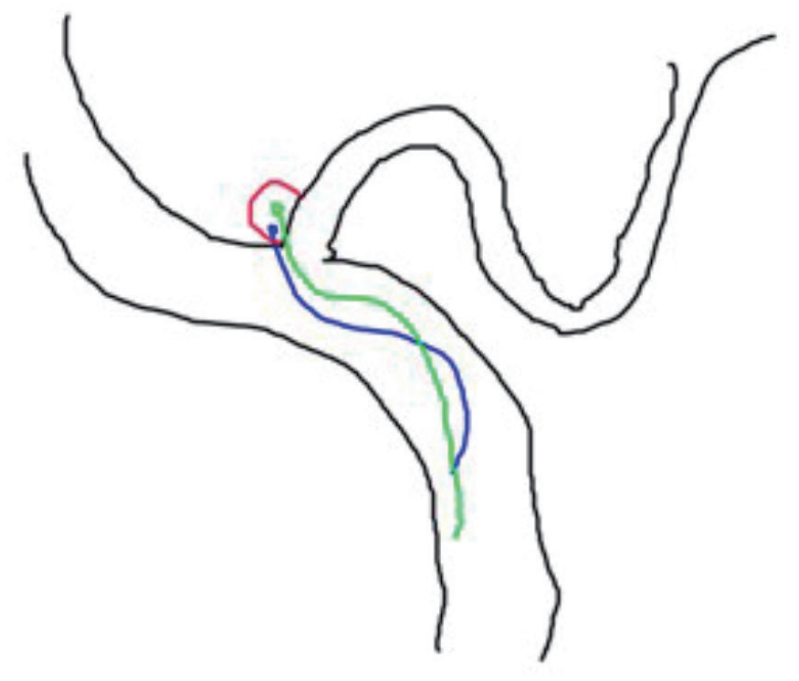

(B)

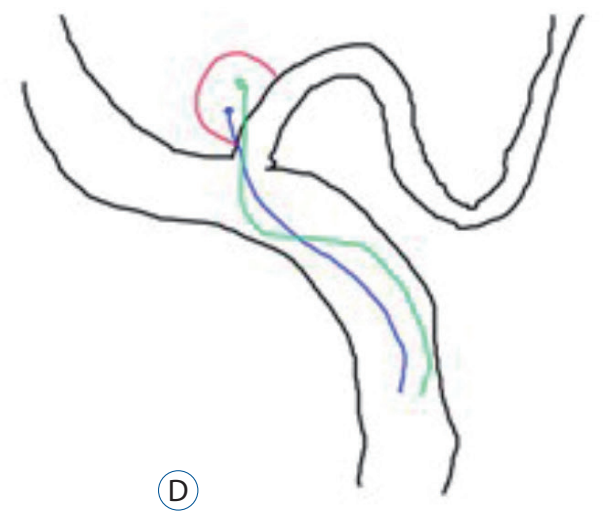

(G)

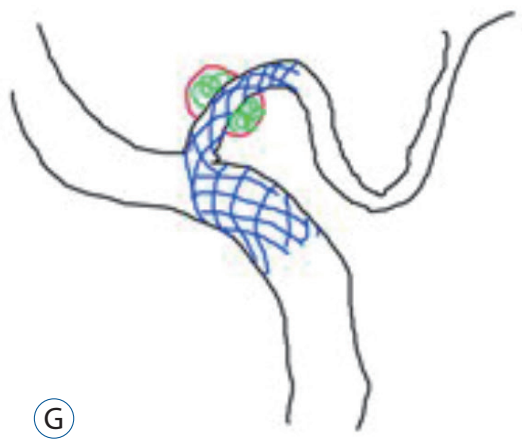

(F)

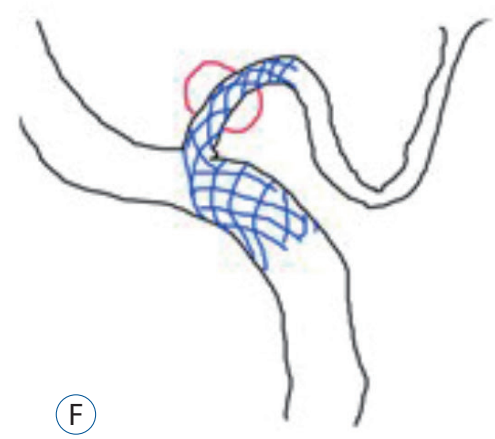

Fig. a relative large neck or compromised the parent artery, an additional microcatheter may be useful for preventing the coil from coming out to the parent artery (B-D). In fusiform aneurysms or suspicious dissecting aneurysm, coil embolization can be performed with a stent-assisted technique (E-G). 

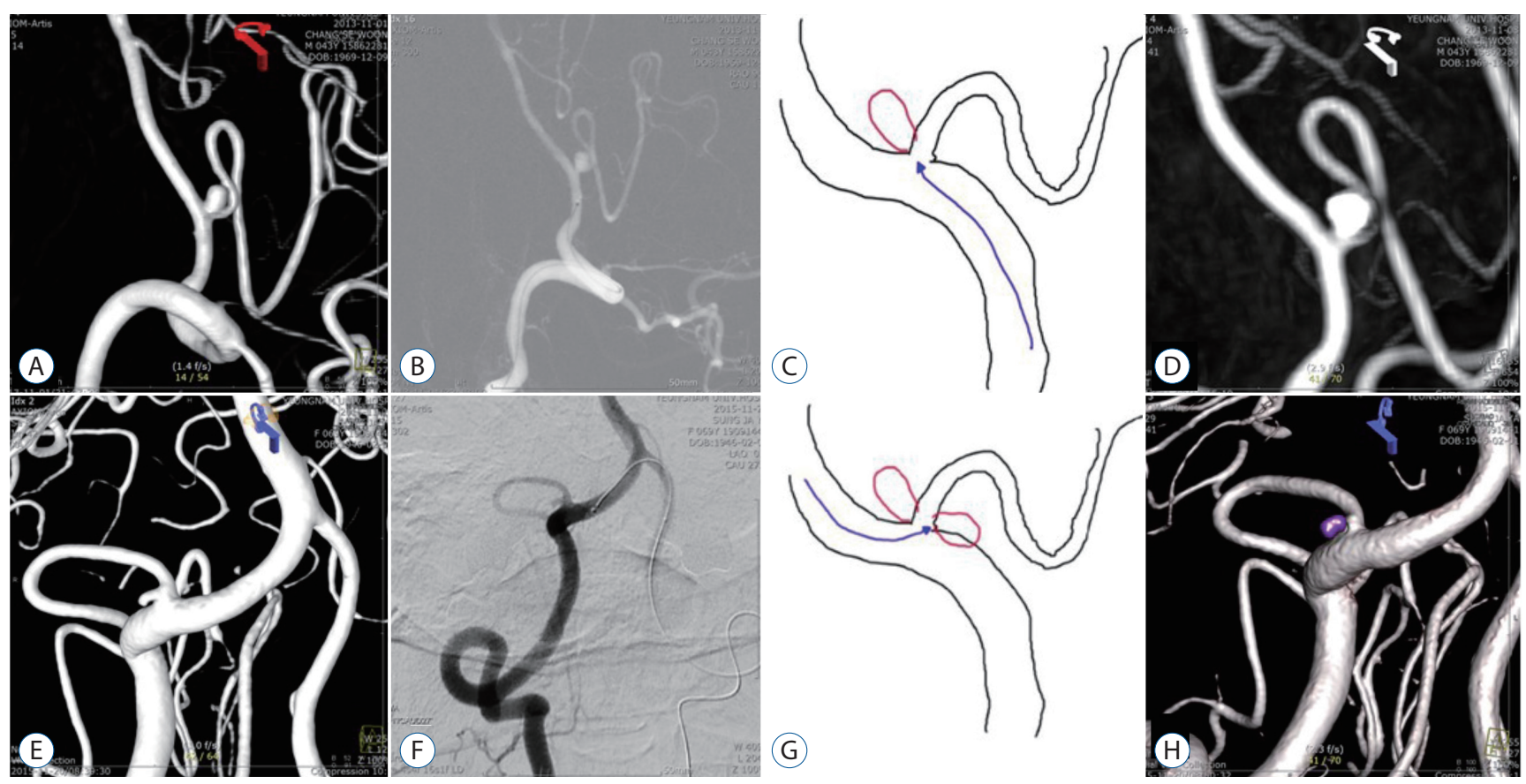

Fig. 4. The 3-dimensional rotational digital subtraction angiogram shows a superior directed proximal PICA aneurysm, arising according the bloodstream flow (A). With the ipsilateral VA approach, the aneurysm is obliterated with a single microcatheter (B-D). The other 3-dimensional angiogram shows the inferolateral directed aneurysm growing opposite to the normal blood flow (E). The microcatheter is approached via the opposite side VA ( $F$ and G). Complete coil embolization of the aneurysm is achieved with a single microcatheter (H). PICA : posterior inferior cerebellar artery, VA : vertebral artery.
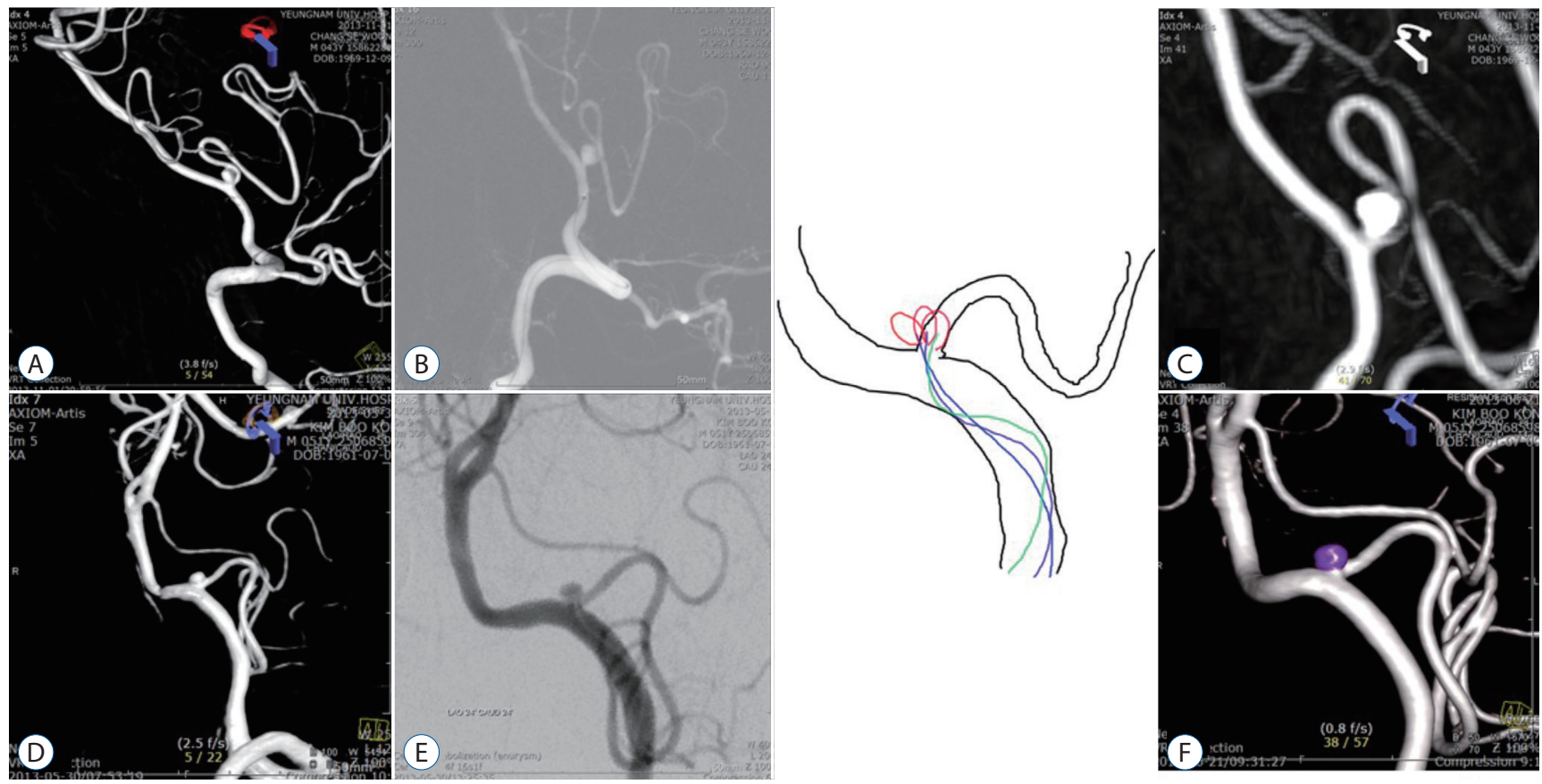

Fig. 5. The 3-dimensional rotational digital subtraction angiogram shows superior directed proximal PICA aneurysms arising according to the bloodstream flow (A and D). In case A, the angle between the aneurysm and the VA is lower than that in case D. After making a loose S-shaped microcatheter with steam, complete coil obliteration was achieved with a single microcatheter (B, C). Case D has a larger angle between the aneurysm and the VA; thus, a more angled S-shaped microcatheter with a double catheter technique was used (D-F). PICA : posterior inferior cerebellar artery, VA : vertebral artery. 


\section{Case 2 : choosing the microcatheter and coil em- bolization technique}

A loose-S-shaped microcatheter was formed using steam and placed in the aneurysm because the angle between the parent artery and the aneurysm was not large (Fig. 5A-C). The preshaped $S$ was placed in the aneurysm because of the large angle between the parent artery and the aneurysm (Fig. 5D-F). Two microcatheters were used to put the coil in the aneurysm because the neck was large and shorter (Fig. 5D-F).

\section{Case 3 : choosing the microcatheter and coil embolization technique}

In a case of an aneurysm completely invading the parent artery, we used two microcatheter techniques to prevent coil protrusion to the parent artery (Fig. 6A). Preshaped 90 and J microcatheters were placed in the aneurysm; then, two pieces of coil were used to perform coil embolization while maintaining the patency of the parent artery (Fig. $6 \mathrm{~B}$ and C).

\section{DISCUSSION}

SAH caused by aneurysmal rupture in the proximal posterior circulation requires first-aid treatment in many cases because of the high likelihood of rebleeding and, consequently, a high morbidity rate ${ }^{3)}$. In particular, SAH is even more infrequently caused by proximal PICA aneurysms ${ }^{5}$. The lesions can be treated with craniotomy followed by clipping, as well as with endovascular coil embolization. Surgical treatment using craniotomy and clipping is difficult to perform in the posterior circulation because of insufficient experience owing to the small number of cases, deep location, and high incidence of hematoma at the rupture site. In addition, it is surrounded by important blood vessels and lower cranial nerves ${ }^{2)}$. Another type of treatment is endovascular coil embolization, which can be performed through existing blood vessels, making it easier to approach lesions than craniotomy and brings about relatively good effects, though in only a few cases ${ }^{6}$. However, although the number of aneurysmal SAH due to ruptured proximal PICA is small, the prevalence is known to be approximately $0.5 \%$ to $2 \%{ }^{6}$. Thus, if a physician has less experience, endovascular coil embolization could also be difficult. In real clinical practice, coil embolization of these lesions is still challenging and has high risk of procedural rupture and procedure-related morbidity ${ }^{6}$. However, because of the high risk of rebleeding, aneurysmal SAH requires emergency treatment. In such an emergency, physicians do not have sufficient time for discussion and consultation with their colleagues in most cases. As the morphology of the aneurysm and its relationship to the parent artery differ among cases, the treatment plan also differs. Therefore, in many cases, such aneurysm poses a challenge in emergency proximal PICA coil embolization. However, having a plan for deciding the approach route, the device, and coil embolization technique for any of these uncommon lesions may be useful in the treatment of SAH.
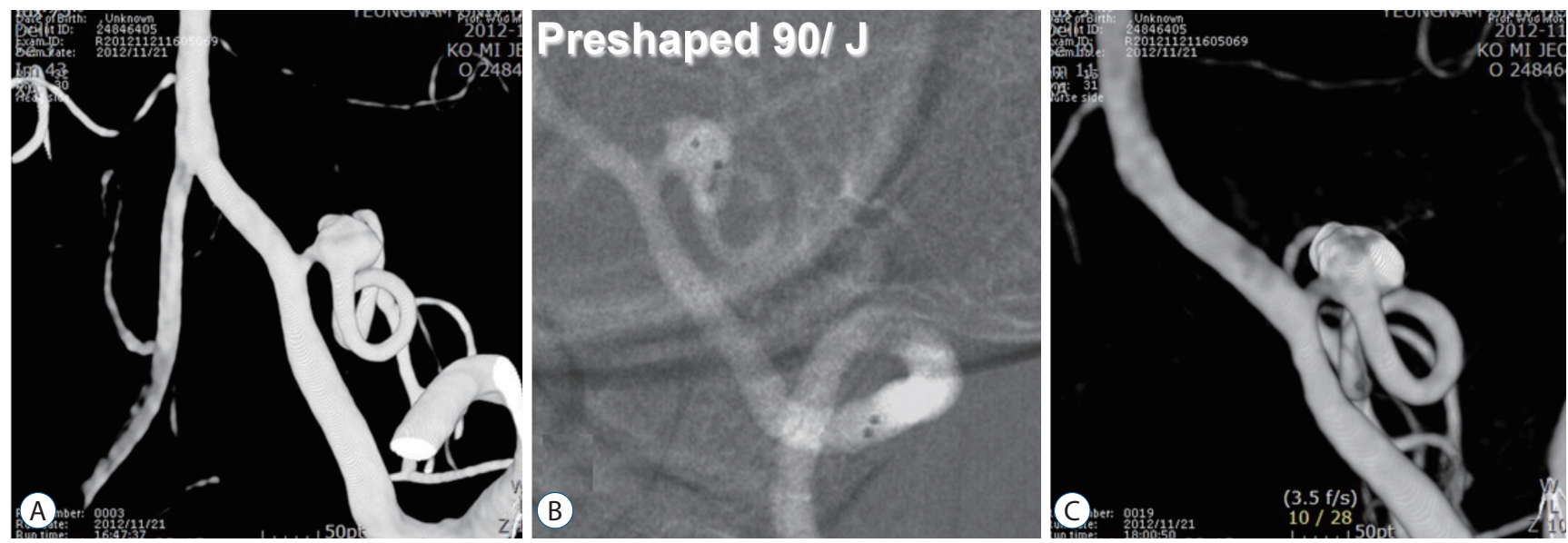

Fig. 6. The 3-dimensional rotational digital subtraction angiogram shows an abnormal dilatation of the proximal PICA. The aneurysm arises at the proximal PICA, and the parent artery is compromised by the aneurysm neck (A). After two microcatheters are inserted in the aneurysm, the frame coil is made simultaneously with two pieces of coil while maintaining patency of the parent artery (B and C). PICA : posterior inferior cerebellar artery. 


\section{CONCLUSION}

Coil embolization of aneurysmal SAH due to ruptured proximal PICA aneurysm is still challenging. However, if an appropriate coiling plan is in place, it could be treated well. In performing acute-phase coil embolization, it is crucial to determine the correlation between the aneurysm and the parent artery and to choose a microcatheter that can make the approach easier and be kept more stable while coiling. Using another microcatheter or a stent, if necessary, is expected to enable successful application of coil embolization even for lesions that failed to be treated with the same technique.

\section{CONFLICTS OF INTEREST}

The authors have no financial conflicts of interest.

\section{INFORMED CONSENT}

Informed consent was obtained from all individual participants included in this study.

\section{- Acknowledgments}

This work was supported by the 2014 Yeungnam University Research Grant.

\section{References}

1. Al-khayat $\mathrm{H}$, Al-Khayat $\mathrm{H}$, Beshay J, Manner D, White J : Vertebral artery-posteroinferior cerebellar artery aneurysms: clinical and lower cranial nerve outcomes in 52 patients. Neurosurgery 56 : 2-10; discussion 11, 2005

2. Bohnstedt BN, Ziemba-Davis M, Edwards G, Brom J, Payner TD, Leipzig TJ, et al. : Treatment and outcomes among 102 posterior inferior cerebellar artery aneurysms: a comparison of endovascular and microsurgical clip ligation. World Neurosurg 83 : 784-793, 2015

3. Connolly ES Jr, Rabinstein AA, Carhuapoma JR, Derdeyn CP, Dion J, Higashida RT, et al. : Guidelines for the management of aneurysmal subarachnoid hemorrhage: a guideline for healthcare professionals from the American Heart Association/American Stroke Association. Stroke 43 : 1711-1737, 2012

4. Jeon SG, Kwon DH, Ahn JS, Kwun BD, Choi CG, Jin SC : Detachable coil embolization for saccular posterior inferior cerebellar artery aneurysms. J Korean Neurosurg Soc 46 : 221-225, 2009

5. Park JS, Lee TH, Seo EK, Cho YJ : Aneurysms of distal posterior inferior cerebellar artery. J Korean Neurosurg Soc 44 : 205-210, 2008

6. Peluso JP, van Rooij WJ, Sluzewski M, Beute GN, Majoie CB : Posterior inferior cerebellar artery aneurysms: incidence, clinical presentation, and outcome of endovascular treatment. AJNR Am J Neuroradiol 29 : $86-90,2008$ 